\title{
RESEARCH
}

Open Access

\section{Centiloid cut-off values for optimal agreement between PET and CSF core AD biomarkers}

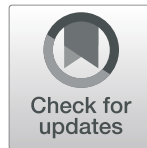

Gemma Salvadó ${ }^{1}$, José Luis Molinuevo 1,2,3*, Anna Brugulat-Serrat ${ }^{1}$, Carles Falcon ${ }^{1,4}$, Oriol Grau-Rivera', Marc Suárez-Calvet ${ }^{1}$, Javier Pavia ${ }^{4,5,6}$, Aida Niñerola-Baizán ${ }^{5}$, Andrés Perissinotti ${ }^{5}$, Francisco Lomeña ${ }^{5}$, Carolina Minguillon ${ }^{1,2}$, Karine Fauria ${ }^{1,2}$, Henrik Zetterberg ${ }^{7,8,9,10}$, Kaj Blennow ${ }^{7,8}$, Juan Domingo Gispert ${ }^{1,3,4^{*}}$ and for the Alzheimer's Disease Neuroimaging Initiative, for the ALFA Study

\begin{abstract}
Background: The Centiloid scale has been developed to standardize measurements of amyloid PET imaging. Reference cut-off values of this continuous measurement enable the consistent operationalization of decisionmaking for multicentre research studies and clinical trials. In this study, we aimed at deriving reference Centiloid thresholds that maximize the agreement against core Alzheimer's disease (AD) cerebrospinal fluid (CSF) biomarkers in two large independent cohorts.

Methods: A total of 516 participants of the ALFA+ Study $(N=205)$ and ADNI $(N=311)$ underwent amyloid PET imaging $\left(\left[{ }^{18} \mathrm{~F}\right]\right.$ flutemetamol and $\left[{ }^{18} \mathrm{~F}\right]$ florbetapir, respectively) and core AD CSF biomarker determination using Elecsys ${ }^{\circledR}$ tests. Tracer uptake was quantified in Centiloid units (CL). Optimal Centiloid cut-offs were sought that maximize the agreement between PET and dichotomous determinations based on CSF levels of A $\beta_{42}$, tTau, pTau, and their ratios, using pre-established reference cut-off values. To this end, a receiver operating characteristic analysis (ROC) was conducted, and Centiloid cut-offs were calculated as those that maximized the Youden's J Index or the overall percentage agreement recorded.

Results: All Centiloid cut-offs fell within the range of 25-35, except for CSF A $\beta_{42}$ that rendered an optimal cut-off value of $12 \mathrm{CL}$. As expected, the agreement of tau/A $\beta_{42}$ ratios was higher than that of CSF A $\beta_{42}$. Centiloid cut-off robustness was confirmed even when established in an independent cohort and against variations of CSF cut-offs.

Conclusions: A cut-off of $12 \mathrm{CL}$ matches previously reported values derived against postmortem measures of $\mathrm{AD}$ neuropathology. Together with these previous findings, our results flag two relevant inflection points that would serve as boundary of different stages of amyloid pathology: one around $12 \mathrm{CL}$ that marks the transition from the absence of pathology to subtle pathology and another one around $30 \mathrm{CL}$ indicating the presence of established pathology. The derivation of robust and generalizable cut-offs for core AD biomarkers requires cohorts with adequate representation of intermediate levels.
\end{abstract}

Trial registration: ALFA+ Study, NCT02485730

ALFA PET Sub-study, NCT02685969

Keywords: AD pathophysiology, Biomarker concordance, Threshold, Positivity, Preclinical, Early detection, Positron emission tomography, Phosphorylated tau, Biomarker categorization

\footnotetext{
* Correspondence: jlmolinuevo@barcelonabeta.org;

jdgispert@barcelonabeta.org

${ }^{1}$ Barcelonaßeta Brain Research Center (BBRC), Pasqual Maragall Foundation,

Wellington 30, 08005 Barcelona, Spain

Full list of author information is available at the end of the article
}

(c) The Author(s). 2019 Open Access This article is distributed under the terms of the Creative Commons Attribution 4.0 International License (http://creativecommons.org/licenses/by/4.0/), which permits unrestricted use, distribution, and

reproduction in any medium, provided you give appropriate credit to the original author(s) and the source, provide a link to the Creative Commons license, and indicate if changes were made. The Creative Commons Public Domain Dedication waiver (http://creativecommons.org/publicdomain/zero/1.0/) applies to the data made available in this article, unless otherwise stated. 


\section{Introduction}

Aggregation of $\beta$-amyloid $(A \beta)$ is a neuropathological hallmark of Alzheimer disease (AD) and occurs decades before the onset of clinical symptoms occur $[1,2]$. Both amyloid positron emission tomography (PET) and cerebrospinal fluid (CSF) $\mathrm{A} \beta_{42}$ measurement are established biomarkers of $A \beta$ deposition that highly correlate with post-mortem [3, 4] and brain biopsy findings [5] and serving as in vivo proxies of AD pathological findings that can be assessed in vivo. They are included as part of the biological definition of AD in the recent NIA-AA 2018 research framework [6] for the definition of preclinical stages of AD [7] and as well as inclusion criteria in clinical trials [8]. CSF A $\beta_{42}$ and amyloid PET show a high degree of agreement [9-19], even though they probably measure two different pools of amyloid. While the signal detected by amyloid PET may reflect fibrillary amyloid [20], the decrease of CSF $A \beta_{42}$ levels more likely reflects both fibrillar and non-fibrillar $A \beta$ deposits. Another difference is that CSF $\mathrm{A} \beta_{42}$ may become abnormal before amyloid PET $[21,22]$, while amyloid PET has been suggested to be superior for grading early symptomatic AD stages [19].

For diagnostic purposes, three ${ }^{18}$ F-labelled PET radiotracers have been granted marketing authorizations and are being used: $\left[{ }^{18} \mathrm{~F}\right]$ flutemetamol (Vizamyl; GE Healthcare), $\left[{ }^{18} \mathrm{~F}\right]$ florbetaben (Neuraceq; Life Molecular Imaging), and $\left[{ }^{18} \mathrm{~F}\right]$ florbetapir (Amyvid, Eli Lilly). In the clinical setting, PET scans are visually read by trained specialists and are categorized as either positive or negative [23]. For quantitative purposes, the three different tracers show considerable variability when measured using the typical standardized update value ratios (SUVRs). To improve the comparability of the retention measurements across tracers, the Centiloid method has been proposed [24]. This method linearly scales the measurement of a particular tracer from 0 to 100 scale, where ' 0 ' represents the average uptake in young controls and ' 100 ' corresponds to the average uptake in typical $\mathrm{AD}$ patients at the dementia stage. To apply the Centiloid conversion, reference datasets, quantification pipelines, and regions of interest and reference are available publically from http://www.gaain.org/ centiloid-project. When expressed in Centiloids (CL), optimal thresholds for positivity against visual reads typically fall within the range between 25 and 35 CL [25-27].

The applicability of CSF $A \beta_{42}$ determinations with enzyme-linked immunosorbent assays (ELISAs) has been limited by several preanalytical and analytical factors, resulting in lot-to-lot and between-laboratory variability. These issues are expected to be improved by the availability of certified reference materials [28], and the problem with analytical variation is expected to be overcome with fully automated systems, such as the novel Elecsys ${ }^{\circ}$ CSF immunoassay [29]. Using this system, core AD biomarkers in CSF have been compared to A $\beta$ PET $[22,30,31]$ and a
CSF cut-off against PET visual read has been established by receiver operating characteristic (ROC) analysis and validated against an independent sample. The resulting areas under the curve (AUC) of CSF $A \beta_{42}$ against $A \beta$ PET visual read ranged from 0.85 to 0.92 . Interestingly, in these studies, tau $/ \mathrm{A} \beta_{42}$ ratios showed a higher agreement against PET visual reading (AUCs 0.94-0.96) than CSF $A \beta_{42}$ alone.

During the last years, investigators have started interventions before the onset of clinical symptoms, when $A \beta_{42}$ changes are detectable using CSF and amyloid PET biomarkers [32-34]. Amyloid positivity is often recognized as the earliest detectable pathophysiological abnormality in AD. Typically, positivity has been operationalized as a positive visual read in an amyloid PET scan. Accordingly, quantitative cut-offs for PET imaging, but also for CSF biomarkers, have been derived against visual reads. However, the question remains of whether lower quantitative cut-offs can be used to detect more subtle amyloid alterations with higher sensitivity, but which still provide good specificity. Such cut-offs are critical for the operationalization of preventive interventions like recruiting cognitively unimpaired individuals into prevention clinical trials. Therefore, there is a need to establish sensitive, reliable and generalizable cut-off values for amyloid PET to detect early amyloid deposition and operationalize decision-making in preventive intervention. In addition, visual inspection of PET scans can render both positive and negative reads throughout a wide range of $C L$ values.

Initial studies to find optimal thresholds have been performed in populations recruited from clinical populations. This translates into samples with extreme values of both CSF $A \beta_{42}$ and amyloid PET, which is AD patients with high amyloid load vs normal cognitive with low amyloid load. This approximation results in defining an optimal cut-off based on a population with low number of individuals with intermediate values around putative threshold values, which may hamper rendering. A critical consideration when deriving such cut-offs is to appropriately populate amyloid values around the cut-offs to derive optimal and robust values. On the other hand, as CSF $A \beta_{42}$ levels start changing earlier than the PET signal, derivation of CL cut-off values against CSF in populations with initial amyloid abnormalities brings the opportunity to derive more sensitive, yet robust and generalizable, CL values associated to early amyloid accumulation.

In this study, we aimed at deriving optimal Centiloid threshold values in amyloid PET that maximize the agreement against established thresholds of CSF core AD biomarkers. To this end, we capitalized on the ALFA+ cohort of cognitively unimpaired individuals enriched for risk factors for AD [35], and in order to improve the generalizability of our results, analogous data from the Alzheimer's Disease Neuroimaging Initiative 
(ADNI; http://adni.loni.usc.edu/) was pooled with that originated in the ALFA+ cohort.

\section{Methods \\ Participants}

ALFA+ is a nested longitudinal long-term study of the ALFA (for ALzheimer's and FAmilies) cohort [35]. In brief, the ALFA cohort was established as a research platform to characterize preclinical AD in 2743 cognitively preserved individuals, aged between 45 and 75 years old with increased risk for $\mathrm{AD}$. In the nested ALFA+ study, participants undergo advanced protocols of magnetic resonance imaging (MRI), amyloid PET imaging with $\left[{ }^{18} \mathrm{~F}\right]$ flutemetamol and CSF core AD biomarkers [35]. The first consecutive 205 participants of the ALFA+ study were included in this work.

In order to have generalizable results reflecting the whole AD continuum, 311 participants from ADNI were also included in this study selected according to the following inclusion criteria: (1) AD CSF core biomarkers analysed with the Elecsys ${ }^{\circ}$ tests available, (2) amyloid PET scan acquired in less than a year from CSF collection available, and (3) MRI acquired with a difference from the time of the PET acquisition of less than a year. All ADNI PET images included were acquired with $\left[{ }^{18} \mathrm{~F}\right]$ florbetapir.

\section{CSF preanalytics of ALFA+ participants}

Fresh CSF samples were collected in 15-mL polypropylene tubes (Sarstedt catalog \#62.554), the supernatant aliquoted into $0.5-\mathrm{mL}$ polypropylene tubes (Sarstedt catalog \#72.730.005), and frozen within $2 \mathrm{~h}$ after lumbar puncture. Aliquots were placed into long-term storage boxes and stored at $-80^{\circ} \mathrm{C}$ until shipment on dry ice for analysis.

\section{CSF analyses on ALFA+ and ADNI}

CSF samples were measured using the Elecsys ${ }^{\circ} \beta$ amyloid(1-42) [29], and the Elecsys ${ }^{\oplus}$ phosphotau (181P) and Elecsys ${ }^{\circ}$ total-tau immunoassays for CSF on a cobas e 601 analyzer (software version 05.02) at the Clinical Neurochemistry Laboratory, University of Gothenburg, Sweden (ALFA+) or at the Biomarker Research Laboratory, University of Pennsylvania, USA (ADNI), according to the kit manufacturer's instructions and as described in previous studies [29].

\section{Predefined CSF cut-offs against PET visual read}

The BioFINDER and ADNI CSF AD core biomarker cut-offs were previously determined against amyloid PET visual read classification [22, 30]. ADNI participants were categorized using ADNI-specific CSF thresholds (please see Additional file 1: Table S1). Given that the ALFA+ study shares the same preanalytical and analytical conditions as BioFINDER, we used the same thresholds previously published for BioFINDER to categorize ALFA+ participants (please, see Additional file 1: Table S1).
On the other hand, we used the previously described conversion factor from BioFINDER to ADNI values [30] in order to pool herein the ALFA+ values with those of ADNI (only for figures, not used for CL cut-offs derivation).

\section{Neuroimage acquisition procedures}

Each cohort had its own acquisition protocol. For ALFA+, a T1-weighted MRI and an $\left[{ }^{18} \mathrm{~F}\right]$ flutemetamol PET scan was acquired in all participants (mean time difference 97.1 days; range [14-343]). The T1-weighted 3D-TFE sequence was acquired in a Philips $3 \mathrm{~T}$ Ingenia $\mathrm{CX}$ scanner with a voxel size of $0.75 \times 0.75 \times 0.75 \mathrm{~mm}^{3}$, FOV $240 \times 240 \times 180$ $\mathrm{mm}^{3}$, sagittal acquisition, flip angle $8^{\circ}, \mathrm{TR}=9.9 \mathrm{~ms}, \mathrm{TE}=$ $4.6 \mathrm{~ms}, \mathrm{TI}=900 \mathrm{~ms}$. PET imaging was conducted in a Siemens Biograph mCT, following a cranial CT scan for attenuation correction. Participants were injected with 185 $\mathrm{MBq}$ (range 166.5-203.5 Mbq) of $\left[{ }^{18} \mathrm{~F}\right]$ flutemetamol, and 4 frames of $5 \mathrm{~min}$ each were acquired $90 \mathrm{~min}$ post-injection. Images were reconstructed with an OSEM3D algorithm using 8 iterations and 21 subsets and with point spread function (PSF) and time of flight (TOF) corrections into a matrix size of $1.02 \times 1.02 \times 2.03 \mathrm{~mm}$.

The methods for ADNI PET and MRI acquisition methods are described in more detail elsewhere (http:// adni.loni.usc.edu/methods/documents/). In brief, all PET images were acquired with $\left[{ }^{18} \mathrm{~F}\right]$ florbetapir, which consisted of 4 frames of $5 \mathrm{~min}$ each, acquired at 50 to 70 min post injection. Most of the T1 sequences used for normalization were magnetization-prepared rapid acquisition gradient echo (MPRAGE), acquired with $1.5 \mathrm{~T}$ or $3 \mathrm{~T}$ scanners. All images, ALFA+ and ADNI, were visually inspected for quality control.

\section{Image processing}

All PET images were preprocessed following the Centiloid [24] pipeline using SPM12 (https://www.fil.ion.ucl. ac.uk/spm/software/spm12/). In brief, PET frames were coregistered. Averaged images were then coregistered to corresponding MRI scans. MRIs were then segmented and normalized to the MNI space together with PET images. We calculated the SUVr in MNI space using the target region provided in the GAAIN website (www.gaain.org) and the whole cerebellum as reference region. SUVr values were then transformed to the Centiloid scale as explained in Additional file 1: Supplementary methods.

\section{Statistical analysis}

Demographic characteristics of both cohorts were first compared. $T$ test for independent samples was used with continuous variables and $\chi^{2}$ with dichotomous variables. To be able to directly compare $A \beta_{42}$ measures from both cohorts, we had to transform ADNI data to account for pre-analytical conditions [30]. This transformation was only used to perform scatter plots but not to perform 
any other analysis, as each dataset had their own cut-offs for the CSF biomarkers (Additional file 1: Table S1).

Optimal Centiloid cut-offs were calculated as those that maximized the Youden's J Index (YI) or the overall percentage agreement (OPA; "accuracy"). YI statistic consists of the summation of the sensitivity and specificity [36], and the OPA reflects the percentage of cases with concordant binary classification with CSF and PET. Both were calculated on the pooled ALFA+ and ADNI data as a function of Centiloid values for $A \beta_{42}$, phosphorylated tau (pTau), total tau (tTau), and $p T a u / A \beta_{42}$ and $t T a u / A \beta_{42}$ ratios. For each CSF biomarker as binary outcome, optimal cut-offs were selected as those showing the maximum value of one of the statistics after minimally smoothing true-positive, true-negative, false-positive and false-negative curves using the 'smooth' function in Matlab (v2018b) with the 'lowess' method and a span value of 0.1 .

On top of YI and OPA, we also calculated the positive percentage agreement (PPA, "sensitivity") and negative percentage agreement (NPA, "specificity") and the area under the curve (AUC) of the receiver operating characteristic (ROC) analysis. All 95\% confidence intervals (95\% CI) for all statistics were derived using bootstrapping methods $(n=5000)$.

In order to explore the generalizability of the calculated thresholds, we also derived them in each cohort individually. Robustness of the Centiloid cut-offs were assessed by deriving them against more liberal CSF thresholds (higher for $A \beta_{42}$ and lower for tau, Additional file 1: Table S1). With this new CSF categorization, we wanted to include those participants that are close to the threshold but still classified as negative ("grey zone", [GZ]). To calculate these new CSF thresholds, we add (or subtract) the $10 \%$ of the original threshold, as this slight variation in the CSF thresholds showed helping to reduce false negatives and to be strongly associated with future $A \beta_{42}$ positivity $[37,38]$.

\section{Results \\ Demographic characteristics and CSF and amyloid biomarkers}

Table 1 shows demographics and characteristics of CSF and amyloid PET biomarkers in the two cohorts included, i.e. ALFA+ and ADNI, which have some differences. The ALFA+ cohort has younger participants, more women and significantly less proportion of positive participants on all AD CSF core biomarkers, as expected as it includes only cognitively preserved participants. By contrast, ADNI participants are in more advanced stages of the disease, with higher number of $A P O E-\varepsilon 4$ carriers and higher frequency of positive core AD CSF biomarkers (Table 1). This also translates into a significant difference in both the mean and average of amyloid PET CL values between both cohorts. As shown in Figs. 2a, 3a and 4a, the ALFA+ cohort covers intermediate CSF and CL values, whereas ADNI participants' CSF and CL measures show a more bimodal pattern. Mean and SD values for CSF biomarkers and Centiloid measures can be found in Additional file 1: Table S2. Scatterplots for pTau and tTau can be found in Additional file 1: Figure S1.

\section{Optimal CL cut-offs}

We computed the optimal CL cut-off values to differentiate individuals within the Alzheimer's continuum and controls using the AD CSF core biomarkers as a reference. We performed the analysis with CSF $A \beta_{42}$ alone, pTau $/ \mathrm{A} \beta_{42}$ and $\mathrm{tTau} / \mathrm{A} \beta_{42}$ ratios and also pTau and tTau

Table 1 Demographics and characteristics of CSF biomarkers and PET quantification measures, overall and by cohort. All the characteristics shown in this table were statistically different $(p<0.001)$ between cohorts

\begin{tabular}{|c|c|c|c|}
\hline & $\operatorname{ALL}(n=516)$ & $\operatorname{ALFA}+(n=205)$ & $\mathrm{ADNI}(n=311)$ \\
\hline Age, mean (SD) [range] & $69.13(9.10)$ [50-92] & $61.01(4.85)$ [50-74] & 74.48 (7.07) [56-92] \\
\hline Women, $n(\%)$ & $286(55.4)$ & $134(65.4)$ & $152(48.9)$ \\
\hline Education, years mean (SD) & $14.98(3.37)$ & $13.49(3.58)$ & $15.96(2.82)$ \\
\hline APOE- $\varepsilon 4$ carriers, $n(\%)$ & $260(50.4)$ & $81(39.5)$ & $179(57.6)$ \\
\hline Positive $A \beta_{42}, n(\%)$ & $273(52.9)$ & $60(29.3)$ & $213(68.5)$ \\
\hline Positive pTau, n (\%) & $323(62.6)$ & $56(27.3)$ & $267(85.9)$ \\
\hline Positive tTau, n (\%) & $294(57.0)$ & $50(24.4)$ & $244(78.5)$ \\
\hline Positive pTau/A $\beta_{42}, n(\%)$ & $258(50.0)$ & $24(11.7)$ & $234(75.2)$ \\
\hline Positive $t T a u / A \beta_{42}, n(\%)$ & $246(47.7)$ & $21(10.2)$ & $225(72.3)$ \\
\hline
\end{tabular}


alone. We studied the pooled data from ALFA+ and ADNI cohorts, using cohort-specific CSF thresholds.

Table 2 summarizes the optimal CL values, using the pooled data from ALFA+ and ADNI cohorts, and associated statistical performance against CSF $\mathrm{A} \beta_{42}$, pTau $/ \mathrm{A} \beta_{42}$, $\mathrm{tTau} / \mathrm{A} \beta_{42}$, pTau and tTau. Figure 1 shows the associated ROC curves.

\section{CSF $A \beta_{42}$}

Derivation of Centiloid cut-off against $A \beta_{42}$ is shown in Fig. 2. The resulting cut-offs with this analysis were 12.1 $\mathrm{CL}$ with YI's optimization and 11.6 CL OPA's optimizations, which corresponded with maximum values YI of 0.66 (95\% CI 0.59-0.72) and OPA of 0.83 (95\% CI 0.81$0.86)$, respectively. The corresponding area under the curve for CSF $A \beta_{42}$ was of 0.87 (95\% CI 0.84-0.90). PPA and NPA values for these CL cut-offs are shown in Table 2.

The optimal cut-offs calculated against 10\% variation of the CSF threshold were similar $(\mathrm{CL}=11.1 \mathrm{CL}$ with $\mathrm{OPA}$, 0.85 , and $\mathrm{CL}=12.9$ with YI, 0.70 ; Additional file 1: Table S3 and Figure S2), and the AUC was slightly improved AUC = 0.90 . When CL cut-offs were derived separately in the two independent cohorts, the AUC for CSF $A \beta_{42}$ was higher in the ADNI cohort than in ALFA+ $(0.85$ vs 0.76 Additional file 1: Table S4). The optimal threshold for the ALFA+ cohort were slightly lower to the one found in the pooled analysis $(\mathrm{CL}=5.4$ with $\mathrm{YI}$ and $\mathrm{CL}=10.7$ with OPA, Additional file 1: Table S4 and Figure S3), whereas, for ADNI, the optimal cut-off resulted was significantly higher $(\mathrm{CL}=36.2 \mathrm{CL}$ with $\mathrm{YI}$ and $\mathrm{CL}=33.1$ with $\mathrm{OPA}$, Additional file 1: Table S4 and Figure S4).

\section{CSF tau $/ \mathrm{A} \beta_{42}$ ratios and tau}

Very similar results were found in both tau over $A \beta_{42}$ ratios (Figs. 3 and 4). The optimal cut-off derived with both cohorts merged for $\mathrm{pTau} / \mathrm{A} \beta_{42}$ was $28.8 \mathrm{CL}$ with an AUC of 0.97 [0.96-0.99] with both YI and OPA's maximization and for $\mathrm{tTau} / \mathrm{A} \beta_{42} 29.7 \mathrm{CL}$ with YI's maximization and 30.1 CL with OPA's maximization with an AUC of 0.96 [0.94-0.97] (Table 2).

Unlike for CSF $A \beta_{42}$, optimal thresholds against variations of the CSF cut-offs resulted in a reduced optimal threshold of $\mathrm{CL}=21.4$ for both tau ratios with YI's maximization and $\mathrm{CL}=20.6$ for both tau ratios with OPA's maximization (Additional file 1: Table S3 and Figure S2). When derived in both cohorts separately, thresholds were again slightly lower in the ALFA+ cohort $(\mathrm{CL}=20.0$ and $\mathrm{CL}=24.8$ for pTau ratio and $\mathrm{CL}=20.1$ and $\mathrm{CL}=24.9$ for tTau ratio; Additional file 1: Figure S3 and Table S4) and significantly higher for ADNI $(\mathrm{CL}=34.4$ and $\mathrm{CL}=31.5$ for pTau ratio and $\mathrm{CL}=34.7$ and $\mathrm{CL}=32.5$ for tTau ratio; Additional file 1: Figure S4 and Table S4).

Optimal cut-offs for CSF pTau and tTau were similar to those for the ratios, but with lower AUCs (Table 2).
Meanwhile, tTau cut-offs remained relatively stable when using YI or OPA as criterion $(\mathrm{CL}=28.6$ and $\mathrm{CL}=28.8$, respectively); pTau cut-offs changed highly (29.3 CL with YI vs 18.7 with OPA; Additional file 1: Table S3). The analysis against 10\% CSF variations resulted in similar cut-offs with similar AUCs except for tTau cut-off resulting from OPA's maximization that lowered up to $15.9 \mathrm{CL}$ (Additional file 1: Table S3 and Figure S2). Finally, the behaviour of the cut-offs in the two cohorts separately was quite different with respect to tau over $A \beta_{42}$ ratios and resulted in lower AUCs than the optimal cut-off (Additional file 1: Figure S2, S3 and, Table S4).

\section{Discussion}

In this paper, we sought to calculate the optimal Centiloid cut-off values from amyloid PET data to maximize the agreement against previously established thresholds for positivity on core AD CSF biomarkers. At a first glance, this might be regarded as a circular exercise, since these CSF cut-offs were originally derived to maximally concord with positive visual reading of PET scans. Under this rationale, all resulting Centiloid cut-offs would have be expected to fall in the range that optimally discriminates negative from positive visual reads, which is between 25 and $35 \mathrm{CL}[25,26]$. On the contrary, optimal agreement for CSF $A \beta_{42}$ was observed for a cut-off of $12 \mathrm{CL}$. This seemingly unexpected result can be explained by the clearly non-linear relationship between amyloid PET Centiloids and CSF $A \beta_{42}$, as previously reported [39]. Almost all subjects with CSF A $\beta_{42}$ over $1000 \mathrm{pg} / \mathrm{ml}$ showed Centiloid values below 20, and only for CSF values $<1000 \mathrm{pg} / \mathrm{ml}$, a linear association could be intuited. This nonlinear association makes goodness criteria (both the Youden's Index and the overall percentage agreement) to plateau between 10 and 40 CL (Fig. 2). Under these circumstances, to derive stable optimal cut-offs, it is critical to make use of a test sample comprising both sufficient concordant positive and negative cases as well as a good representation of individuals falling within intermediate amyloid ranges $(10<\mathrm{CL}<40$ and $500<\mathrm{CSF} A \beta_{42}<1000 \mathrm{pg} / \mathrm{ml}$ ). In this study, this was achieved by pooling the ADNI and ALFA+ datasets.

The independent derivation of optimal CSF $A \beta_{42}$ cut-offs in the two cohorts confirmed this rationale. In the ADNI sample, optimal values fell in the expected range of visual reads (between 33 and $36 \mathrm{CL}$ ) whereas the optimal threshold in the ALFA+ sample is closer to that in the pooled sample (between 5 and $11 \mathrm{CL}$ ). This result confirms that the derivation of optimal cut-offs is very sensitive to the recruitment strategy of the reference sample, with clinical ones rendering higher cut-offs than population based ones with a better representation around the range of values where cut-offs are expected to lay. Previous literature has suggested that CSF $A \beta_{42}$ 


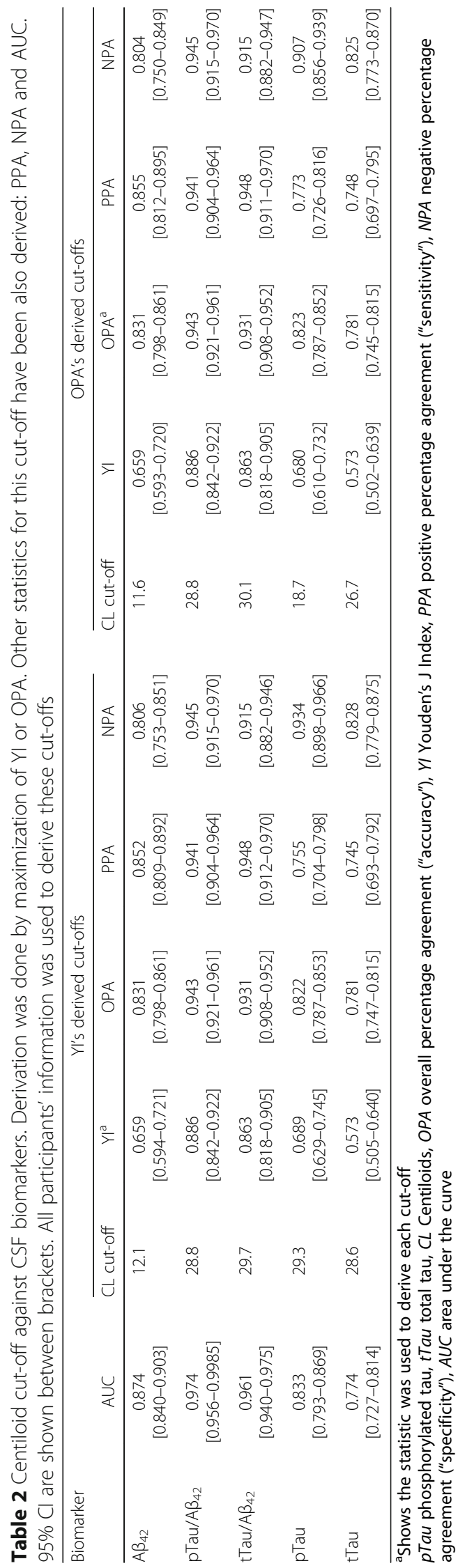




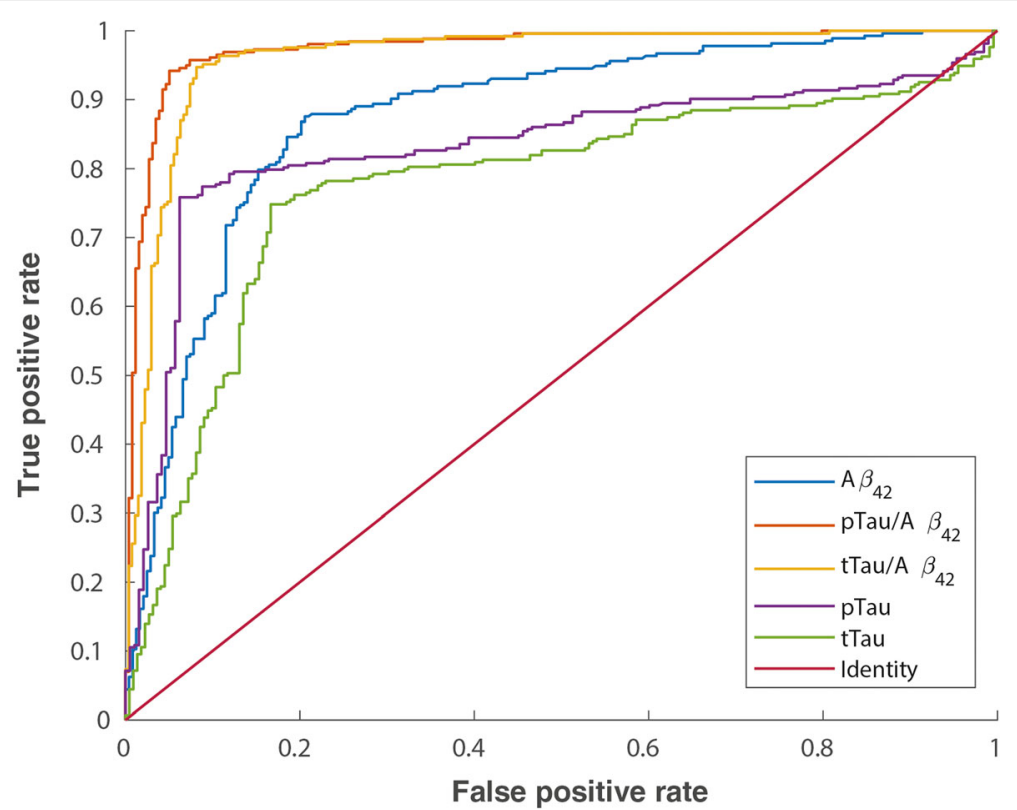

Fig. 1 Summary of ROC curves for Centiloid cut-off derivation against CSF biomarkers. pTau, phosphorylated tau; tTau, total tau; CSF, cerebrospinal fluid; ROC, receiver operating characteristic

values become positive before amyloid PET [21, 22]. This may be related to amyloid PET visual read and SUVRs cut-offs on clinical populations. In these populations, the visual read is performed in patients with either prodromal $\mathrm{AD}$ or dementia due to $\mathrm{AD}$; hence, the amyloid load is supposed to have reached its ceiling. By contrast, the ALFA+ population reflects a cohort of early amyloid accumulators at risk for cognitive impairment; therefore, a positive visual read may be reached when amyloid is still not at its peak. Indeed, the threshold of $12 \mathrm{CL}$ is robust against variations in the cut-off for CSF positivity (Additional file 1: Table S3) as well as if only the ALFA+ dataset is used for its derivation.

Although 12 CL may initially be regarded as a low value for amyloid positivity, it matches recent reports of Centiloid cut-offs against postmortem measures of $\mathrm{AD}$ neuropathology showing that $12.2 \mathrm{CL}$ optimally detected Consortium to Establish a Registry for Alzheimer's Disease (CERAD) moderate-to-frequent neuritic plaques, whereas 24.4 CL identified intermediate-to-high $\mathrm{AD}$ neuropathologic change (ADNC) differences [40]. Another similar study showed that a threshold $<10 \mathrm{CL}$ was optimal for ruling out the presence of amyloid plaques, whereas $\mathrm{CL}>20$ suggests significant amyloid pathology [26]. However, we would like to point out that we did not want to affirm that the CL cut-offs found show amyloid pathology, but only to find those $\mathrm{CL}$ values that maximally agree with those of the CSF core AD biomarkers. The fact that the CL cut-offs derived in this study agree with a previous one done with neuropathological data is only a marker that these values might have an actual biological meaning, more than only a practical one. But this hypothesis should be tested in another work, preferably with longitudinal data.

Unlike CSF $A \beta_{42}$, for tau/CSF $A \beta_{42}$ ratios, the optimal CL cut-offs fell within the expected range (28-30 $\mathrm{CL})$ given the linear relationship between this biomarker and amyloid PET Centiloids. Indeed, tau/CSF $\mathrm{A} \beta_{42}$ ratios showed higher AUC versus amyloid PET Centiloids than CSF $\mathrm{A} \beta_{42}$, in agreement with the previous reports [22, 27, 30, 31]. The higher capacity of tau/CSF $\mathrm{A} \beta_{42}$ ratios to predict Centiloids may be accounted for by two different factors. On the one hand, CSF ratios may provide a more stable measurement than absolute values since they provide an inherent normalization against protein production and release to the CSF, between-individual variations in CSF dynamics, and pre-analytical conditions. Therefore, the lower variability in the CSF ratios may account for better AUCs. On the other hand, CSF A $\beta_{42}$ has been proposed to become abnormal prior to amyloid PET [21, 41]. This fact might stem from the fact that both techniques probe different pools of the amyloid protein. Therefore, the combination of measurements of $A \beta$ with those of tau, a pathological change that is expected to occur later in the $\mathrm{AD}$ continuum [41], might show better agreement with amyloid PET, which is also expected to become abnormal later than CSF $\mathrm{A} \beta_{42}$.

Together with previous studies, the observed thresholds might be useful to flag two different inflection points in preclinical AD stages. A cut-off below $12 \mathrm{CL}$ might be optimal to rule out-amyloid pathology, whereas a cut-off over $29 \mathrm{CL}$ might be denoting established 


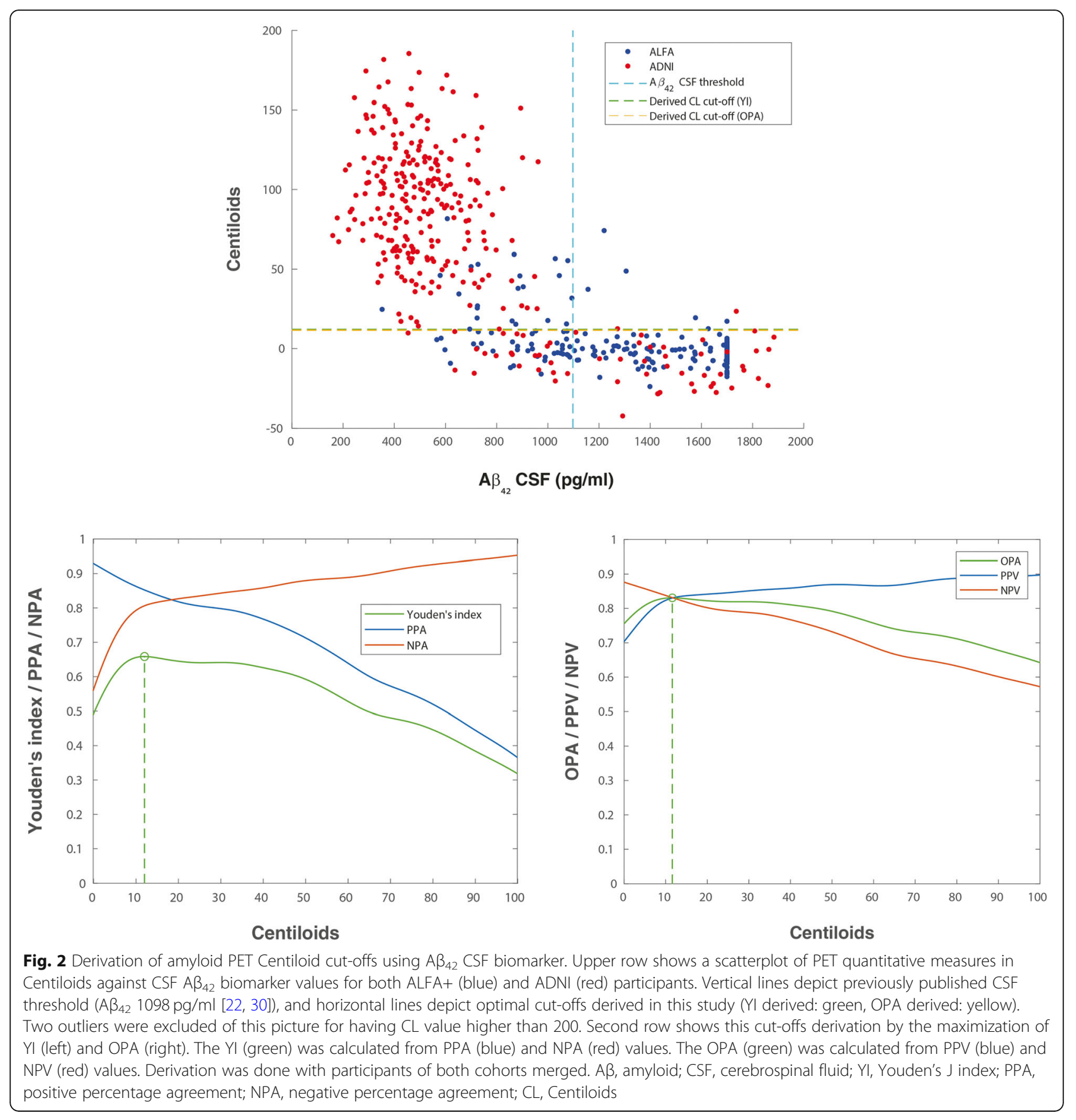

pathology. These kinds of thresholds have typically been used to dichotomize continuous values into two categories for clarity and ease of use. However, alternatives have also been considered and include the score of the severity of each biomarker on a semi-continuous scale as considered, for instance, in the A/T/N scheme [42]. Therefore, an option would be to categorize the full range of variation of biomarker values in three categories: one that excludes any pathology, another intermediate category that would indicate early and developing pathology and a third one that corresponds with established pathology.
Two goodness criteria have been used here to derive optimal cut-offs: the Youden's Index, which balances sensitivity and specificity, and the overall percentage agreement, which is sensitive to the percentages of positives versus negatives in the test sample. Both rendered very similar values and the Centiloid cut-offs proposed here are robust against variations in the threshold values for CSF positivity. Still, the Youden's index showed a more noisy behaviour than the overall percentage agreement, particularly in the analysis of the two individual samples. In order to obtain more robust estimates of 


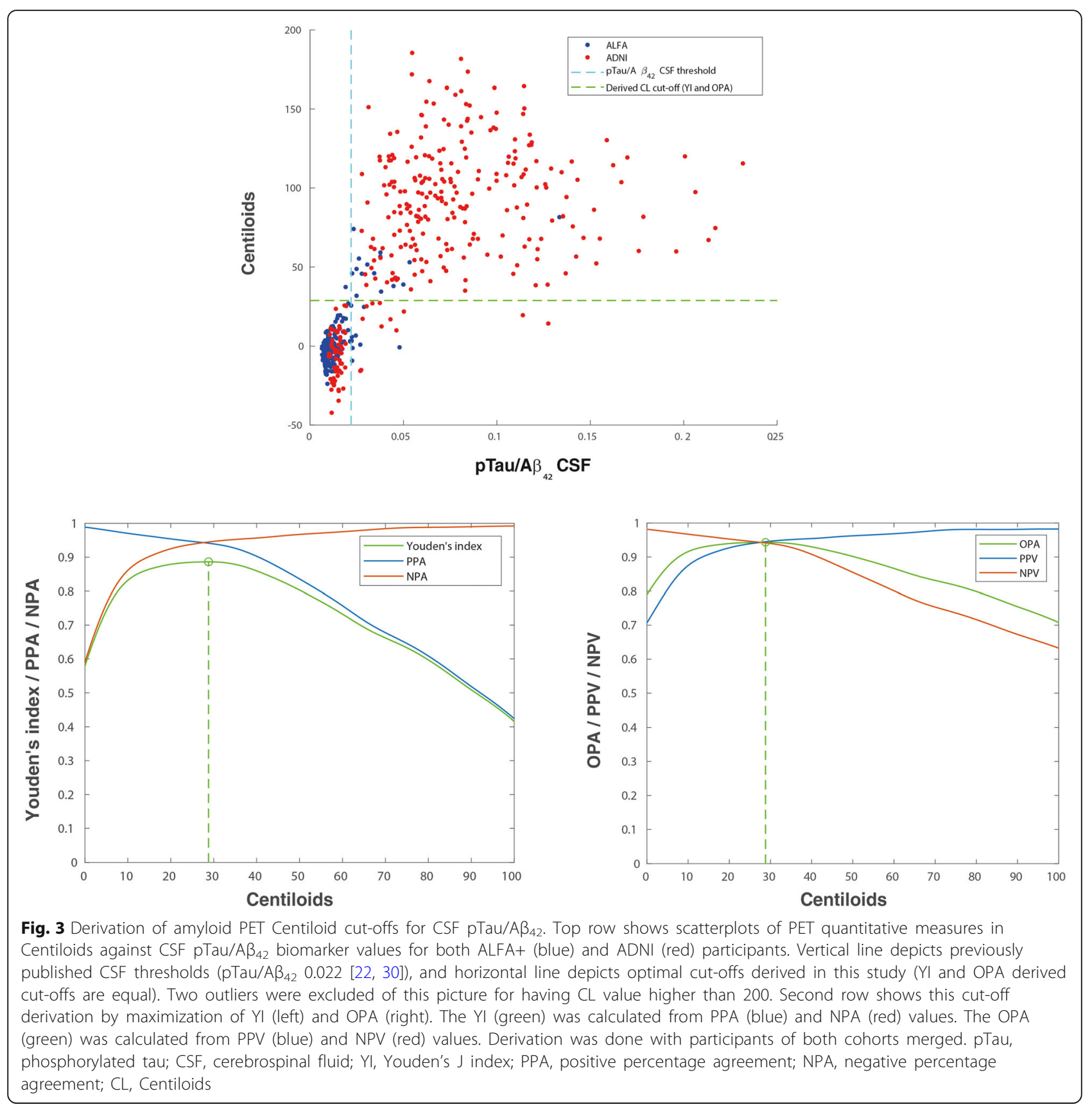

classification performance, more subjects across the full AD continuum would be needed. This is a relevant effect because in previous similar works, the Youden's Index has been typically selected as the reference measurement of agreement [22, 30, 31]. Hansson et al. [30] added reliability measures to performance metrics to derive optimal cut-offs. We handled the noisy behaviour of performance metrics by deriving optimal cut-offs after some minimal smoothing of the data. This approach proved to be efficacious to derive stable cut-offs even in the analysis of the individual cohorts.
Irrespective of the approach to counter the effect of noisy agreement estimates, additional analysis with larger samples might be needed to yield more robust and generalizable cut-offs. To this end, future work will focus on pooling additional samples. In addition to a limited sample size, we rely on the comparability of PET and CSF measures across the two studied samples. While agreement on CSF data is certainly improved with the Elecsys $^{\oplus}$ tests and with the use of the Centiloid method on PET scans, we cannot rule out the presence of a certain degree of sample-dependent bias in the data analysed. Still, when computing the cut-offs solely using the 


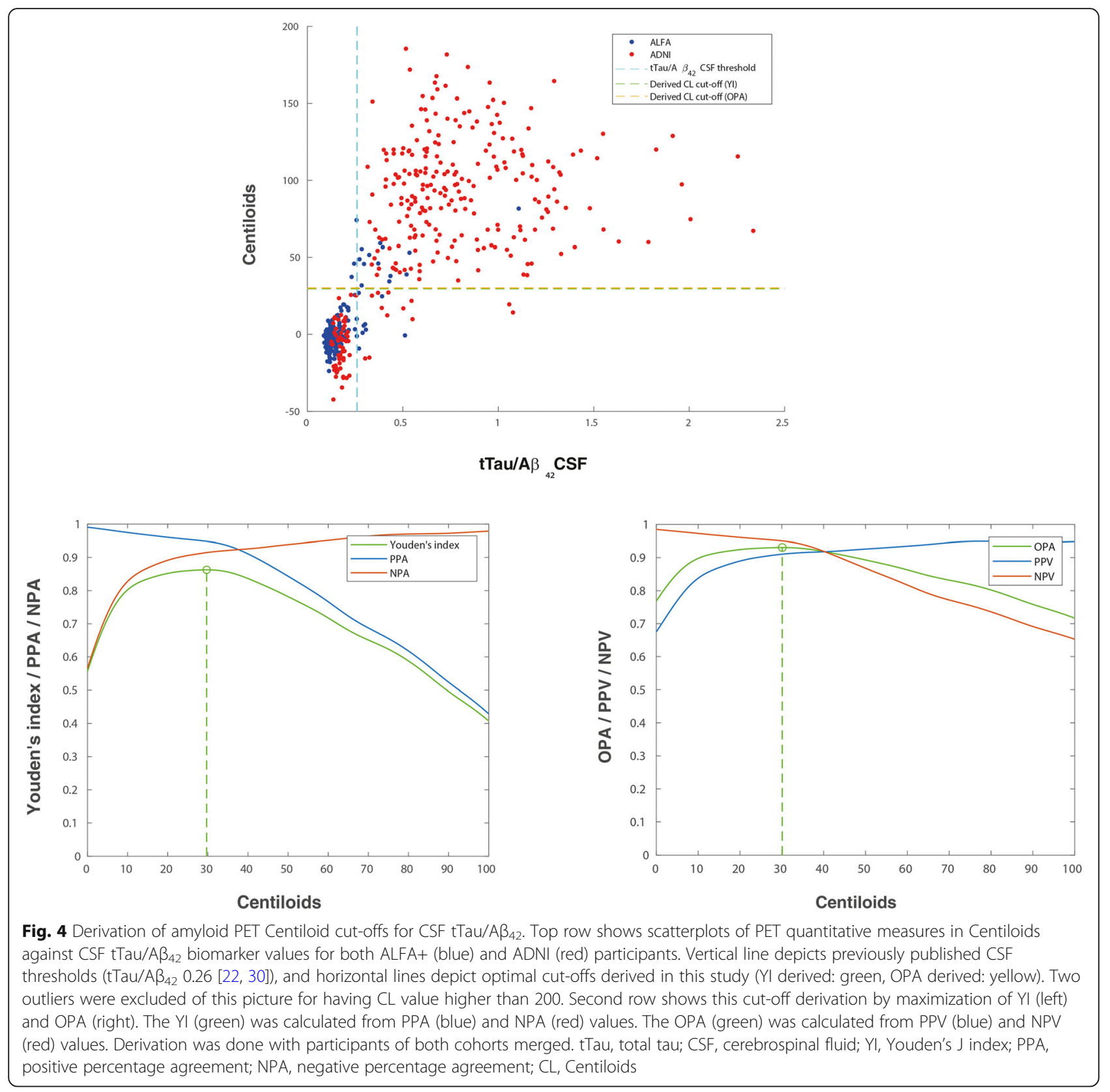

ALFA+ cohort results were very similar, thus suggesting that any remaining bias is small and did not have a significant impact on our results. Another limitation may stem from the somewhat limited sample analysed here may not be sufficient to derive robust generalizable cut-off values. Additional analysis with bigger sample sizes and more amyloid PET tracers that the two used here may overcome this limitation.

In summary, we have derived optimal Centiloid values to maximize the agreement against core AD CSF biomarkers. Regarding $A \beta$, a relatively low value of $12 \mathrm{CL}$ optimally corresponded to CSF $A \beta_{42}$ positivity, in line with Centiloid thresholds derived against post-mortem measures of $\mathrm{AD}$ neuropathology. On the other hand, CSF tau $/ A \beta_{42}$ ratios were better predicted by a higher Centiloid cut-off of $29 \mathrm{CL}$, which is in line with those optimally discriminating positive from negative visual reads on PET scans. In agreement with previous reports, CSF tau $/ A \beta_{42}$ ratios showed a higher capacity to predict amyloid PET Centiloids than CSF $A \beta_{42}$. Overall, our results provide reference values in the Centiloid scale and suggest two relevant inflection points the development of early $\mathrm{AD}$ pathology across the full $\mathrm{AD}$ continuum. 


\section{Additional file}

Additional file 1: Supplementary data including supplementary methods and results. (DOCX $1010 \mathrm{~kb}$ )

\section{Abbreviations}

AD: Alzheimer's disease; ADNC: AD neuropathologic change; ADNI: Alzheimer's Disease Neuroimaging Initiative; ALFA: Alzheimer and Families; AUC: Area under the curve; A $\beta$ : $\beta$-Amyloid; CERAD: Consortium to Establish a Registry for Alzheimer's Disease; Cl: Confidence interval; CL: Centiloid; CSF: Cerebrospinal fluid; ELISA: Enzyme-linked immunosorbent assay; GZ: Grey zone; MPRAGE: Magnetization-prepared rapid acquisition gradient echo; MRI: Magnetic resonance imaging; NPA: Negative percentage agreement ("specificity"); OPA: Overall percentage agreement ("accuracy"), PET: Positron emission tomography; PPA: Positive percentage agreement ("sensitivity"); PSF: Point spread function; pTau: Phosphorylated tau; ROC: Receiver operating characteristic; SUVr: Standardized uptake value ratio; TOF: Time of flight; tTau: Total tau; YI: Youden's J index

\section{Acknowledgements}

This publication is part of the ALFA study (ALzheimer and FAmilies). The authors would like to express their most sincere gratitude to the ALFA project participants, without whom this research would have not been possible.

Authors would like to thank GE Healthcare for kindly providing ${ }^{18} \mathrm{~F}$ flutemetamol doses of ALFA+ participants and Roche Diagnostics International Ltd. for kindly providing the kits for the CSF analysis of ALFA+ participants. Authors would also like to thank Christopher Foley, Christopher Buckley and Mark Battle for their help on the Centiloid calculation. Collaborators of the ALFA study are: Jordi Camí, Raffaele Cacciaglia, Marta Crous-Bou, Carme Deulofeu, Ruth Dominguez, Xavi Gotsens, Nina Gramunt, Laura Hernandez, Gema Huesa, Jordi Huquet, María León, Paula Marne, Tania Menchón, Marta Milà, Grégory Operto, Maria Pascual, Albina Polo, Sandra Pradas, Aleix Sala-Vila, Gonzalo Sánchez-Benavides, Sabrina Segundo, Anna Soteras, Laia Tenas, Marc Vilanova, Natalia Vilor-Tejedor.

Data collection and sharing for this project was funded by the Alzheimer's Disease Neuroimaging Initiative (ADNI) (National Institutes of Health Grant U01 AG024904) and DOD ADNI (Department of Defense award number W81XWH-12-2-0012). ADNI is funded by the National Institute on Aging, the National Institute of Biomedical Imaging and Bioengineering, and through generous contributions from the following: AbbVie, Alzheimer's Association; Alzheimer's Drug Discovery Foundation; Araclon Biotech; BioClinica, Inc.; Biogen; Bristol-Myers Squibb Company; CereSpir, Inc.; Cogstate; Eisai Inc.; Elan Pharmaceuticals, Inc.; Eli Lilly and Company; Eurolmmun; F. Hoffmann-La Roche Ltd and its affiliated company Genentech, Inc.; Fujirebio; GE Healthcare; IXICO Ltd.; Janssen Alzheimer Immunotherapy Research \& Development, LLC.; Johnson \& Johnson Pharmaceutical Research \& Development LLC.; Lumosity; Lundbeck; Merck \& Co., Inc.; Meso Scale Diagnostics, LLC. NeuroRx Research; Neurotrack Technologies; Novartis Pharmaceuticals Corporation; Pfizer Inc.; Piramal Imaging; Servier; Takeda Pharmaceutical Company; and Transition Therapeutics. The Canadian Institutes of Health Research is providing funds to support ADNI clinical sites in Canada. Private sector contributions are facilitated by the Foundation for the National Institutes of Health (www.fnih.org). The grantee organization is the Northern California Institute for Research and Education, and the study is coordinated by the Alzheimer's Therapeutic Research Institute at the University of Southern California. ADNI data are disseminated by the Laboratory for Neuro Imaging at the University of Southern California.

Data used in precreation of this article were obtained from the Alzheimer's Disease Neuroimaging Initiative (ADNI) database (adni.loni.usc.edu). As such the investigators within the ADNI contributed to the design and implementation of ADNI and/or provided data but did not participate in analysis or writing of this report. A complete listing of ADNI investigators can be found at (http://adni.loni.usc.edu/wp-content/uploads/how_to_apply/ ADNI_Acknowledgement_List.pdf).

\section{Funding}

The research leading to these results has received funding from "la Caixa" Foundation (LCF/PR/GN17/10300004) and the Alzheimer's Association and an international anonymous charity foundation through the the TriBEKa Imaging
Platform project. JDG holds a 'Ramón y Cajal' fellowship (RYC-2013-13054). MS-C receives funding from the European Union's Horizon 2020 research and innovation programme under the Marie Sklodowska-Curie action grant agreement No 752310. CM is supported by the Spanish Ministry of Economy and Competitiveness (grant $n^{\circ}$ IEDI-2016-00690). KB holds the Torsten Söderberg Professorship in Medicine at the Royal Swedish Academy of Sciences, and is supported by the Swedish Research Council (\#2017-00915), the Swedish Alzheimer Foundation (\#AF-742881), Hjärnfonden, Sweden (\#FO2017-0243), and a grant (\#ALFGBG-715986) from the Swedish state under the agreement between the Swedish government and the County Councils, the ALF-agreement.

$\mathrm{HZ}$ is a Wallenberg Academy Fellow supported by grants from the Swedish Research Council and the European Research Council.

\section{Availability of data and materials}

The datasets generated and/or analysed during the current study are not publicly available.

\section{Authors' contributions}

All authors listed (GS, JLM, AB-S, CF, OG-R, MS-C, JP, AN, AP, FL, CM, KF, HZ, $\mathrm{KB}$ and JDG) made a substantial contribution to the concept and design, acquisition of data or analysis and interpretation of data; drafted the article or revised it critically for important intellectual content; and approved the final version to be published.

\section{Ethics approval and consent to participate}

The ALFA study and the PET sub-study protocols have been approved by an independent Ethics Committee Parc de Salut Mar Barcelona and registered at Clinicaltrials.gov (ALFA Identifier: NCT02485730; PET sub-study Identifier: NCT02685969). Both studies have been conducted in accordance with the directives of the Spanish Law 14/ 2007, of 3rd of July, on Biomedical Research (Ley 14/ 2007 de Investigación Biomédica).

\section{Consent for publication}

Not applicable.

\section{Competing interests}

$J$ LM is a consultant for the following for-profit companies: Alergan, Roche diagnostics, Genentech, Novartis, Lundbeck, Oryzon, Biogen, Lilly, Janssen, Green Valley, MSD, Eisai, Alector, Raman Health.

Other authors declared no potential conflicts of interest with respect to the research, authorship, and/or publication of this article.

\section{Publisher's Note}

Springer Nature remains neutral with regard to jurisdictional claims in published maps and institutional affiliations.

\section{Author details}

${ }^{1}$ Barcelonaßeta Brain Research Center (BBRC), Pasqual Maragall Foundation, Wellington 30, 08005 Barcelona, Spain. ${ }^{2}$ CIBER Fragilidad y Envejecimiento Saludable (CIBERFES), Madrid, Spain. ${ }^{3}$ Universitat Pompeu Fabra, Barcelona, Spain. ${ }^{4} \mathrm{CIBER}$ de Bioengeniería, Biomateriales y Nanomedicina, Madrid, Spain. ${ }^{5}$ Nuclear Medicine Department, Hospital Clínic, Barcelona, Spain. ${ }^{6}$ Instititut $\mathrm{d}^{\prime}$ Investigacions Biomèdiques August Pi i Sunyer, Barcelona, Spain. ${ }^{7} \mathrm{Clinical}$ Neurochemistry Laboratory, Sahlgrenska University Hospital, Mölndal, Sweden. ${ }^{8}$ Department of Psychiatry and Neurochemistry, Institute of Neuroscience and Physiology, Sahlgrenska Academy at University of Gothenburg, Sahlgrenska University Hospital, Mölndal, Sweden. ${ }^{9}$ Department of Neurodegenerative Disease, UCL Institute of Neurology, Queen Square, London, UK. ${ }^{10}$ UK Dementia Research Institute at UCL, London, UK.

Received: 10 January 2019 Accepted: 27 February 2019

Published online: 21 March 2019

\section{References}

1. Villemagne $V L$, Burnham S, Bourgeat P, Brown B, Ellis KA, Salvado O, et al. Amyloid $\beta$ deposition, neurodegeneration, and cognitive decline in sporadic Alzheimer's disease: a prospective cohort study. Lancet Neurol. 2013;12:357-67. 
2. Vos SJB, Xiong PC, Visser PJ, Ms MSJ, Hassenstab J, Grant EA, et al. Preclinical Alzheimer's disease and its outcome: a longitudinal cohort study. Lancet Neurol. 2013;12:957-65.

3. Shaw LM, Vanderstichele H, Knapik-Czajka M, Clark CM, Aisen PS, Petersen $\mathrm{RC}$, et al. Cerebrospinal fluid biomarker signature in Alzheimer's disease neuroimaging initiative subjects. Ann Neurol. 2009;65:403-13.

4. Ikonomovic MD, Buckley CJ, Heurling K, Sherwin P, Jones PA, Zanette M, et al. Post-mortem histopathology underlying $\beta$-amyloid PET imaging following flutemetamol F 18 injection. Acta Neuropathol Commun. 2016;4:130.

5. Seppälä TT, Nerg O, Koivisto AM, Rummukainen J, Puli L, Zetterberg H, et al. CSF biomarkers for Alzheimer disease correlate with cortical brain biopsy findings. Neurol. 2012;78:1568 LP-1575.

6. Jack CR, Bennett DA, Blennow K, Carrillo MC, Dunn B, Haeberlein SB, et al NIA-AA Research Framework: toward a biological definition of Alzheimer's disease. Alzheimers Dement. 2018;14:535-62.

7. Sperling RA, Aisen PS, Beckett LA, Bennett DA, Craft S, Fagan AM, et al. Toward defining the preclinical stages of Alzheimer's disease. Alzheimers Dement. 2011;7:280-92.

8. Sperling RA, Mormino EC, Johnson KA. The evolution of preclinical Alzheimer's disease: implications for prevention trials. Neuron. 2014;84:608-22.

9. Fagan AM, Mintun MA, Mach RH, Lee SY, Dence CS, Shah AR, et al. Inverse relation between in vivo amyloid imaging load and cerebrospinal fluid Abeta 42 in humans. Ann Neurol. 2006:59:512-9.

10. Fagan AM, Mintun MA, Shah AR, Aldea P, Roe CM, Mach RH, et al. Cerebrospinal fluid tau and ptau181increase with cortical amyloid deposition in cognitively normal individuals: implications for future clinical trials of Alzheimer's disease. EMBO Mol Med. 2009;1:371-80.

11. Grimmer T, Riemenschneider M, Förstl H, Henriksen G, Klunk WE, Mathis CA, et al. Beta amyloid in Alzheimer's disease: increased deposition in brain is reflected in reduced concentration in cerebrospinal fluid. Biol Psychiatry. 2009;65:927-34.

12. Forsberg A, Almkvist $\mathrm{O}$, Engler $\mathrm{H}$, Wall A, Langstrom B, Nordberg A. High PIB retention in Alzheimers disease is an early event with complex relationship with CSF biomarkers and functional parameters. Curr Alzheimer Res. 2010;7:56-66.

13. Jagust WJ, Landau SM, Shaw LM, Trojanowski JQ, Koeppe RA, Reiman EM, et al. Relationships between biomarkers in aging and dementia. Neurol. 2009;73:1193-9.

14. Koivunen J, Pirttilä T, Kemppainen N, Aalto S, Herukka SK, Jauhianen AM, et al. PET amyloid ligand [11C]PIB uptake and cerebrospinal fluid $\beta$-amyloid in mild cognitive impairment. Dement Geriatr Cogn Disord. 2008;26:378-83.

15. Landau SM, Lu M, Joshi AD, Pontecorvo M, Mintun MA, Trojanowski JQ, et al. Comparing positron emission tomography imaging and cerebrospinal fluid measurements of $\beta$-amyloid. Ann Neurol. 2013;74:826-36.

16. Tolboom N, van der Flier WM, Yaqub M, Boellaard R, Verwey NA, Blankenstein MA, et al. Relationship of cerebrospinal fluid markers to $11 \mathrm{C}$ PiB and 18F-FDDNP binding. J Nucl Med. 2009;50:1464-70.

17. Weigand SD, Vemuri P, Wiste HJ, Senjem ML, Pankratz VS, Aisen PS, et al. Transforming cerebrospinal fluid A 42 measures into calculated Pittsburgh compound $B$ units of brain A 3 amyloid. Alzheimers Dement. 2011;7:133-41.

18. Palmqvist $\mathrm{S}$, Zetterberg $\mathrm{H}$, Mattsson $\mathrm{N}$, Johansson $\mathrm{P}$, Minthon L, Blennow $\mathrm{K}$, et al. Detailed comparison of amyloid PET and CSF biomarkers for identifying early Alzheimer disease. Neurol. 2015;85:1240-9.

19. Palmqvist S, Zetterberg H, Blennow K, Vestberg S, Andreasson U, Brooks DJ, et al. Accuracy of brain amyloid detection in clinical practice using cerebrospinal fluid $\beta$-amyloid 42: a cross-validation study against amyloid positron emission tomography. JAMA Neurol. 2014;11:1282-9.

20. Mathis CA, Lopresti BJ, Ikonomovic MD, Klunk WE. Small-molecule PET tracers for imaging proteinopathies; 2017.

21. Palmqvist $\mathrm{S}$, Mattsson N, Hansson O. Cerebrospinal fluid analysis detects cerebral amyloid- $\beta$ accumulation earlier than positron emission tomography. Brain. 2016;139:1226-36.

22. Schindler SE, Gray JD, Gordon BA, Xiong C, Batrla-Utermann R, Quan M, et al. Cerebrospinal fluid biomarkers measured by Elecsys assays compared to amyloid imaging. Alzheimers Dement. 2018;14(11):1460-469.

23. Morris E, Chalkidou A, Hammers A, Peacock J, Summers J, Keevil S. Diagnostic accuracy of $18 \mathrm{~F}$ amyloid PET tracers for the diagnosis of Alzheimer's disease: a systematic review and meta-analysis. Eur J Nucl Med Mol Imaging. 2016;43:374-85.
24. Klunk WE, Koeppe RA, Price JC, Benzinger TL, Devous MD, Jagust WJ, et al The Centiloid project: standardizing quantitative amyloid plaque estimation by PET. Alzheimers Dement. 2015;11:1-15.e4.

25. Rowe CC, Doré V, Jones G, Baxendale D, Mulligan RS, Bullich S, et al. 18FFlorbetaben PET beta-amyloid binding expressed in Centiloids. Eur J Nucl Med Mol Imaging. 2017:44:2053-9.

26. Rowe CC, Amadoru S, Dore V, McLean CL, Hinton F, Shepherd C, et al. Correlation of amyloid PET in Centiloid units with neuropathological findings in Alzheimer's disease. J Nucl Med. 2018;59:482.

27. Leuzy A, Chiotis K, Hasselbalch SG, Rinne JO, De Mendonça A, Otto M, et al. Pittsburgh compound B imaging and cerebrospinal fluid amyloid- $\beta$ in a multicentre European memory clinic study. Brain. 2016;139:2540-53.

28. Kuhlmann J, Andreasson U, Pannee J, Bjerke M, Portelius E, Leinenbach A, et al. CSF A 1-42- an excellent but complicated Alzheimer's biomarker - a route to standardisation. Clin Chim Acta. 2017;467:27-33.

29. Bittner T, Zetterberg H, Teunissen CE, Ostlund RE, Militello M, Andreasson U, et al. Technical performance of a novel, fully automated electrochemiluminescence immunoassay for the quantitation of $\beta$-amyloid (1-42) in human cerebrospinal fluid. Alzheimers Dement. 2016;12:517-26.

30. Hansson O, Seibyl J, Stomrud E, Zetterberg H, Trojanowski JQ, Bittner T, et al. CSF biomarkers of Alzheimer's disease concord with amyloid- $\beta$ PET and predict clinical progression: a study of fully automated immunoassays in BioFINDER and ADNI cohorts. Alzheimers Dement. 2018;14(11):1470-481.

31. Shaw LM, Waligorska T, Fields L, Korecka M, Figurski M, Trojanowski JQ, et al. Derivation of cutoffs for the Elecsys ${ }^{\oplus}$ amyloid $\beta$ (1-42) assay in Alzheimer's disease. Alzheimers Dement Diagnosis Assess Dis Monit. 2018;10:698-705.

32. Ritchie CW, Molinuevo JL, Truyen L, Satlin A, Van der Geyten S, Lovestone S. Development of interventions for the secondary prevention of Alzheimer's dementia: the European Prevention of Alzheimer's Dementia (EPAD) project. Lancet Psychiatry. 2016;3:179-86.

33. Weninger S, Carrillo MC, Dunn B, Aisen PS, Bateman RJ, Kotz JD, et al. Collaboration for Alzheimer's prevention: principles to guide data and sample sharing in preclinical Alzheimer's disease trials. Alzheimers Dement. 2016;12:631-2.

34. McDade E, Bateman RJ. Stop Alzheimer's before it starts. Nature. 2017:547: $153-5$.

35. Molinuevo JL, Gramunt N, Gispert JD, Fauria K, Esteller M, Minguillon C, et al The ALFA project: a research platform to identify early pathophysiological features of Alzheimer's disease. Alzheimers Dement Transl Res Clin Interv. 2016;2:82-92

36. Youden WJ. Index for rating diagnostic tests. Cancer. 1950;3:32-5.

37. Molinuevo JL, Blennow K, Dubois B, Engelborghs S, Lewczuk P, PerretLiaudet A, et al. The clinical use of cerebrospinal fluid biomarker testing for Alzheimer's disease diagnosis: a consensus paper from the Alzheimer's biomarkers standardization initiative. Alzheimers Dement. 2014;10:808-17.

38. Mattsson N, Insel PS, Donohue $M$, Jagust W, Sperling R, Aisen P, et al. Predicting reduction of cerebrospinal fluid $\beta$-amyloid 42 in cognitively healthy controls. JAMA Neurol. 2015:72:554-60.

39. Toledo JB, Bjerke M, Da X, Landau SM, Foster NL, Jagust W, et al. Nonlinear association between cerebrospinal fluid and florbetapir F-18 $\beta$-amyloid measures across the spectrum of Alzheimer disease. JAMA Neurol. 2015;72:571-81.

40. La Joie R, Ayakta N, Seeley WW, Borys E, Boxer AL, DeCcarli C, et al. Multisite study of the relationships between antemortem [11C]PIB-PET Centiloid values and postmortem measures of Alzheimer's disease neuropathology. Alzheimers Dement. 2018;15(2):205-216

41. Jack CR, Knopman DS, Jagust WJ, Petersen RC, Weiner MW, Aisen PS, et al. Tracking pathophysiological processes in Alzheimer's disease: An updated hypothetical model of dynamic biomarkers. Lancet Neurol. 2013;12(2):207-16.

42. Jack CR, Bennett DA, Blennow K, Carrillo MC, Feldman HH, Frisoni GB, et al. $A / T / N$ : an unbiased descriptive classification scheme for Alzheimer disease biomarkers. Neurol. 2016;87:539-47. 